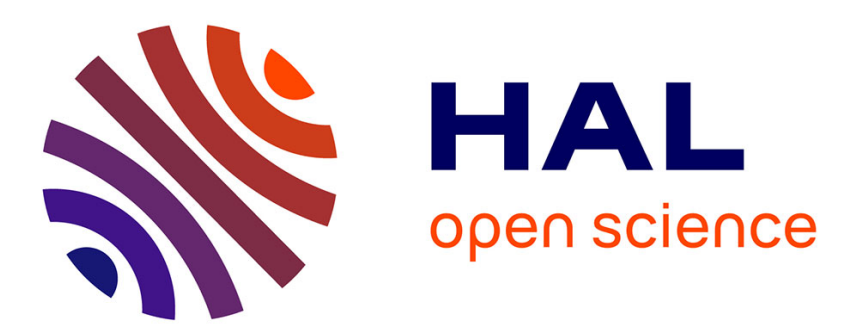

\title{
Quad-Rotor Switching Control: An Application for the Task of Path Following
}

Luis-Rodolfo Garcia Carrillo, Gerardo Flores, Guillaume Sanahuja, Rogelio

Lozano

\section{- To cite this version: \\ Luis-Rodolfo Garcia Carrillo, Gerardo Flores, Guillaume Sanahuja, Rogelio Lozano. Quad-Rotor Switching Control: An Application for the Task of Path Following. American Control Conference (ACC 2012), Jun 2012, Montréal, Canada. pp.4637-4642. hal-00776113}

\section{HAL Id: hal-00776113 \\ https://hal.science/hal-00776113}

Submitted on 15 Jan 2013

HAL is a multi-disciplinary open access archive for the deposit and dissemination of scientific research documents, whether they are published or not. The documents may come from teaching and research institutions in France or abroad, or from public or private research centers.
L'archive ouverte pluridisciplinaire HAL, est destinée au dépôt et à la diffusion de documents scientifiques de niveau recherche, publiés ou non, émanant des établissements d'enseignement et de recherche français ou étrangers, des laboratoires publics ou privés. 


\title{
Quad-Rotor Switching Control: An Application for the Task of Path Following
}

\author{
L.R. García Carrillo ${ }^{\dagger}$, G. Flores ${ }^{\dagger}$, G. Sanahuja ${ }^{\dagger}$, and R. Lozano ${ }^{\star}$
}

\begin{abstract}
The problem of vision-based road following using a quad-rotor is addressed. The objective consists of estimating and tracking a road without a priori knowledge of such path. For this purpose, two operational regions are defined: one for the case when the road is detected, and the other one for when it is not. A switching between imaging and inertial sensors measurements allows estimating the required vehicle's parameters in both regions. Also, for dealing with both aforementioned cases, a switching control strategy which stabilizes the vehicle's lateral position is proposed. The performance of the proposed switching methodologies is tested in real time experiments.
\end{abstract}

\section{INTRODUCTION}

This work is motivated by the theory of switching systems and the need of developing effective UAVs controllers and state estimators, not only for attitude and position stabilization, but also for successfully executing a more complex predefined mission. The problem of stabilizing a quad-rotor using visual feedback has been constantly addressed, see for example [1], [2], [3]. It has been previously shown that PD controllers work efficiently for stabilizing the UAV's attitude dynamics [4]. Also, PD controllers have proved to be robust enough for similar applications than the one being addressed in the present research [5]. In both previous approaches, the imaging system was used for estimating the vehicle's translational dynamics. However, those strategies are not robust solutions for dealing with external disturbances causing that the imaging system losses temporarily the artificial landmarks used for obtaining the vehicle's states. In [6], the authors propose a switching strategy for estimating the states of a UAV equipped with imaging, inertial and air data sensing systems, capable of dealing with instants of time when the visual detection of the landmark fails. Following a similar reasoning, the implementation of different controllers (and therefore different gains) is required by the fact that, the use of a PD controller in the position dynamics is not enough for attenuating the disturbances caused by, for example, winds gusts [7]. The switching system approach has been previously implemented to solve UAVs related problems. In [8], the authors present the analysis and control of a VTOL aircraft at simulation level.

This work was partially supported by the Mexican National Council for Science and Technology (CONACYT) and the Institute for Science \& Technology of Mexico City (ICyTDF)

$\dagger$ are with the Heudiasyc UMR 6599 Laboratory, University of Technology of Compiègne, France. L.R. García Carrillo (email: lgarciac@hds.utc.fr), G. Flores (email: gfloresc@hds.utc.fr), G. Sanahuja (email: gsanahuj@hds.utc.fr)

* is with the Heudiasyc UMR 6599 Laboratory, UTC CNRS France and LAFMIA UMI 3175, Cinvestav, México. (email: rlozano@hds.utc.fr)
By using a common Lypunov function, the stability of the complete system, divided in three modes, is proved.

This paper addresses the problem of autonomously perform a road following mission using a quad-rotor UAV, over a road having smooth curves. Estimating and controlling the vehicle's relative position, orientation and velocity with respect to (w.r.t.) the road, are the subjects of interest. A camera allows estimating the vehicle's heading angle w.r.t. the road's longitudinal orientation, as well as the lateral distance that the vehicle must correct in order to navigate exactly over the road. A switching control strategy for estimation and tracking between two operational regions is defined: one for the situation when the road is in the camera field of view (FOV), and the other for the situation when it is not. The system's stability is proved not only in the two regions, but also in the switching boundaries between them. The performance of the switching control is tested in real time experiments, demonstrating the effectiveness of the proposed approach.

The paper is organized as follows. Section II presents the problem statement. The methods proposed to estimate the vehicle's states are shown in Section III. The switching control strategy and the stability proof are presented in Section IV. Section V presents the quad-rotor experimental platform. The performance of the UAV during real-time experiments is shown in Section VI. Finally, Section VII presents some conclusions.

\section{Problem Statement}

The vehicle considered is equipped with inertial sensors, an ultrasonic range finder and a calibrated camera. The inertial system provides the vehicle's angular dynamics, allowing the development of a control strategy for attitude stabilization. The ultrasonic range finder points downwards, directly measuring the altitude of the aerial vehicle during flight. The camera is installed on-board pointing downwards, in a manner that the plane formed by the helicopter's $\left(x_{h}, y_{h}\right)$ plane and the camera's $\left(y_{c}, x_{c}\right)$ image plane are parallel and have the same orientation. The camera and the aerial vehicle move together as a rigid body, therefore, using the image provided by the camera, vision-based strategies could be implemented to estimate the vehicle's states required for the navigation mission.

The road-following task can be detailed as follows. First the vehicle performs an autonomous take-off, reaching a desired altitude $z_{d}$ over the road. At this point, the heading of the vehicle, expressed by $\psi$, is driven to yield a parallel positioning between the helicopter $x$-axis (represented by $x_{h}$ ) and the 
longitudinal direction of the road (expressed by $x_{r}$ ). The helicopter's forward speed, expressed by $\dot{x}$, is kept to a constant value while the distance between $x_{h}$ and $x_{r}$, expressed by $e_{y}$, is regulated and kept to a minimum value, achieving a flight path well aligned and centered w.r.t. the road. Finally, the quad-rotor is required to land autonomously in a position near the end of the road.

The main goal is to design a road following strategy based on computer vision and switching control, with the purpose of stabilizing the vehicle's altitude, heading angle and lateral position ( $z, \psi$ and $y$ states respectively) w.r.t. the road, while traveling at constant forward speed $(\dot{x}=c)$ in presence of external disturbances.

\section{States ESTIMATION USING Vision}

Consider that the vehicle is flying over a road having smooth curves, which is represented in the camera's image as a group of straight and parallel lines, see Fig. 1. A straight line in the image can be seen as a segment of infinite length and whose center of gravity belongs to the straight line [9]. Based on the Hough transform method for line detection, a straight line is represented as $\rho=x \cos \theta+y \sin \theta$. The center of gravity $\left(x_{g}, y_{g}\right)$ of each straight line detected can be computed as $x_{g}^{i}=\cos (\theta) \rho$ and $y_{g}^{i}=\sin (\theta) \rho$, where the super-index $i$ stands for the line $i$. It is possible to assign initial and final bounds to the line. Let's define $\left(x_{I}^{i}, y_{I}^{i}\right)$ as the initial point of the line, located in a place below the image's lower margin, and let $\left(x_{F}^{i}, y_{F}^{i}\right)$ be the final point of the line, located in a place above the image's upper margin. If the line has a parameter $\rho>0$, the coordinates $\left(x_{I}^{i}, y_{I}^{i}\right)$ and $\left(x_{F}^{i}, y_{F}^{i}\right)$ will be defined as

$$
\begin{array}{ccc}
x_{I}^{i}=x_{g}+\Gamma(-\sin (\theta)) \quad ; & y_{I}^{i}=y_{g}+\Gamma \cos (\theta) \\
x_{F}^{i}=x_{g}-\Gamma(-\sin (\theta)) \quad ; & y_{F}^{i}=y_{g}-\Gamma \cos (\theta)
\end{array}
$$

where $\Gamma$ is a known constant defining the line's bounds. When $\rho<0$, the point $\left(x_{F}^{i}, y_{F}^{i}\right)$ will be computed as in equation (1) while the point $\left(x_{I}^{i}, y_{I}^{i}\right)$ will be computed as in equation (2). The set of parallel lines extracted from the road's projection on the image are grouped yielding just a single average line. This average line will uniquely represent the road in the image

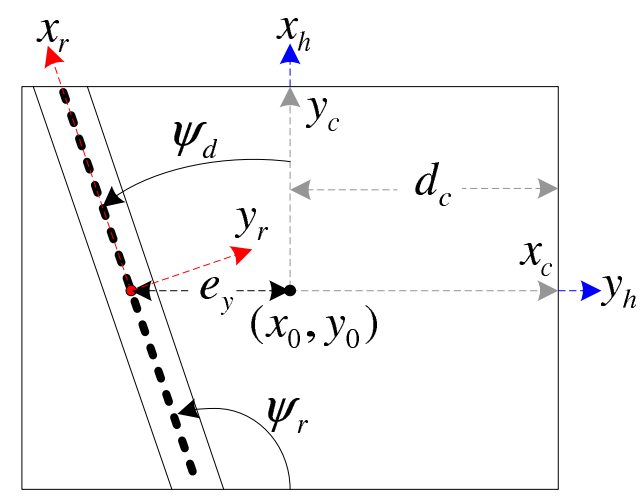

Fig. 1. The camera's view: projection of a road with longitudinal axis $x_{r}$. with a simple pair of initial and final coordinates

$$
\left(x_{I}=\frac{x_{I}^{i}}{i}, y_{I}=\frac{y_{I}^{i}}{i}\right) \quad ; \quad\left(x_{F}=\frac{x_{F}^{i}}{i}, y_{F}=\frac{y_{F}^{i}}{i}\right)
$$

where $i$ is the number of lines grouped together, $\left(x_{I}, y_{I}\right)$ represents the initial (lowermost) road coordinate and $\left(x_{F}, y_{F}\right)$ represents the final (uppermost) road coordinate.

\section{A. Computing the heading angle}

The angle $\psi_{r}$ between the camera's $x_{c}$ axis and the point given by $\left(x_{F}, y_{F}\right)$ can be computed using $\left(x_{I}, y_{I}\right)$ and $\left(x_{F}, y_{F}\right)$ in the two argument function atan2 as $\psi_{r}=$ $\operatorname{atan} 2\left(y_{F}-y_{I}, x_{F}-x_{I}\right)$. The angle $\psi_{r}$ is used for obtaining the desired heading angle $\psi_{d}$ that will align the vehicle's $x$ axis $\left(x_{h}\right)$ with the road's longitudinal axis $\left(x_{r}\right)$, see Fig. 1. $\psi_{d}$ will finally be expressed as $\psi_{d}=\psi_{r}+\frac{\pi}{2}$, where the therm $\frac{\pi}{2}$ is added to adjust $\psi_{d}$ to a value of zero when $\psi_{r}$ is aligned vertically with $x_{h}$.

\section{B. Computing the relative lateral position}

Consider an image-based distance $e_{x}^{c}$ along the camera's $x_{c}$ axis. $e_{x}^{c}$ is defined between the road's center of gravity projection $\left(x_{g}, y_{g}\right)$ and the vehicle's center of gravity projection $\left(x_{0}, y_{0}\right)$, see Fig. 1 . In the case when $x_{I}>x_{F}$, one has

$$
e_{x}^{c}=\left(\frac{x_{I}-x_{F}}{2}+x_{F}\right)-\frac{c_{w}}{2}
$$

where $c_{w}$ represents the width of the image in pixels. In the case when $x_{I}<x_{F}, x_{I}$ must be replaced by $x_{F}$ and viceversa. The lateral position of the aerial vehicle w.r.t. the road can be estimated from $e_{x}^{c}$ as

$$
e_{y}=z \frac{e_{x}^{c}}{\alpha_{x}}
$$

where $z$ represents the distance existing between the helicopter and the road (altitude), and $\alpha_{x}$ represents the camera's focal length in terms of pixel dimensions in the $x_{c}$ direction.

\section{Translational velocities}

Consider a camera-vehicle arrangement moving in a 3dimensional space with respect to a rigid scene. The optical flow coming from an image point $\left(x_{i}, y_{i}\right)$ is composed of a translational part $T_{O F}$ and a rotational part $R_{O F}$, see [5] for details. When computing optical flow, all the $\left(x_{i}, y_{i}\right)$ image coordinates are known, furthermore, they all share the same movement. By using all the tracked features, a mean value for the optical flow can be expressed as

$$
\begin{aligned}
& O \bar{F}_{x}=\bar{V}_{O F_{\mathrm{x}}}+K_{x} \bar{V}_{O F_{\mathrm{z}}}+\bar{R}_{O F_{x}} \\
& O \bar{F}_{y}=\bar{V}_{O F_{\mathrm{y}}}+K_{y} \bar{V}_{O F_{\mathrm{z}}}+\bar{R}_{O F_{y}}
\end{aligned}
$$

where $O \bar{F}_{x}$ and $O \bar{F}_{y}$ are the means of the optical flow sensed in the image coordinate system, $\bar{V}_{O F_{\mathrm{z}}}$ represents the relative depth and $K_{x}$ and $K_{y}$ are known scale factors depending on intrinsic parameters of the camera. Following a similar approach approach than the one presented in [5], the rotational optical flow terms $\bar{R}_{O F_{x}}$ and $\bar{R}_{O F_{y}}$ are compensated and the pseudo-speeds $\left(\bar{V}_{O F_{x}}, \bar{V}_{O F_{y}}, \bar{V}_{O F_{z}}\right)$ are deduced, finally yielding $-z \frac{\bar{V}_{O F_{x}}}{\alpha_{x}}=\dot{x},-z \frac{\bar{V}_{O F_{y}}}{\alpha_{y}}=\dot{y}$ and $z \bar{V}_{O F_{z}}=\dot{z}$. 
D. Computing heading and lateral position when the road is not detected

The computation of the vehicle's desired heading angle and lateral position are compromised by the sensitivity of the Hough transform method for lines detection. Furthermore, it is not possible to estimate such parameters when the the vehicle is flying over a region where the road is out of the camera's FOV. Therefore, the results presented in Section III must be extended to deal with such situations. Let's define a binary signal $s:[0, \infty) \rightarrow\{0,1\}$ as

$$
s(t):= \begin{cases}0 & \text { no line detection at time } t \\ 1 & \text { camera detects line at time } t\end{cases}
$$

allowing to switch between two different methods for computing the vehicle's states. Lets define the time event $T_{s 0}$ as the time when the binary signal changes from 1 to 0 , and $T_{s 1}$ the time event when the binary signal changes from 0 to 1 . Consider the desired heading angle $\psi_{d}$. Equation (8) allows us to define

$$
\psi_{s}(t)=s(t) \psi_{d}(t)+(1-s(t)) \psi_{I M U}\left(T_{s 0}\right)
$$

where $\psi_{d}(t)$ is obtained from the imaging system, $\psi_{I M U}\left(T_{s 0}\right)$ is the IMU's heading angle measurement at the time event $T_{s 0}$, and $\psi_{s}(t)$ represents the desired heading angle that will be used in the helicopter controller. Equation (9) allows switching from a $\psi_{s}(t)$ obtained from the imaging algorithm and a $\psi_{s}(t)$ defined by the IMU's heading angle measurement at the time event when the road was not detected in the image. Consider now the vehicle's lateral position computation when the road is out of sight. Equation (8) allows to compute

$$
e_{y s}(t)=s(t) e_{y}(t)+(1-s(t))\left(e_{y}\left(T_{s 0}\right)+\Sigma_{s}\right)
$$

with $\Sigma_{s}=\int_{T_{s 0}}^{T_{s 1}} \dot{y}(t) d(t)$. Here $e_{y}(t)$ is given by equation (5), $e_{y}\left(T_{s 0}\right)$ is the lateral position measurement at the time event $T_{s 0}$, and $\dot{y}(t)$ represents the vehicle's lateral velocity obtained from optical flow. Equation (10), allows switching between two different approaches for estimating the vehicle's a lateral position w.r.t. the road. In the first approach, lateral position is directly obtained from the detection of the road line in the camera's image (equation (5)). The second approach makes use of the road's position at time event $T_{s 0}$ in combination with the integral of the translational velocity $\dot{y}$ during the time the road is not being detected by the vision algorithm, i.e., during a time bounded by time events $T_{s 0}$ and $T_{s 1}$.

\section{CONTROL}

A normalized mathematical model for the quad-rotor is implemented [10]:

$$
\begin{aligned}
\ddot{x} & =-u_{1}(\cos \phi \sin \theta \cos \psi+\sin \phi \sin \psi) \\
\ddot{y} & =-u_{1}(\cos \phi \sin \theta \sin \psi-\sin \phi \cos \psi) \\
\ddot{z} & =1-u_{1}(\cos \phi \cos \theta) \\
\ddot{\theta} & =u_{2} \\
\ddot{\phi} & =u_{3} \\
\ddot{\psi} & =u_{4}
\end{aligned}
$$

such model can be written in a state-space form by introducing $\dot{X}=\left(x_{1}, \ldots, x_{6}\right)^{T}=(x, \dot{x}, y, \dot{y}, z, \dot{z})^{T}$ and $\dot{Z}=$ $\left(z_{1}, \ldots, z_{6}\right)^{T}=(\theta, \dot{\theta}, \phi, \dot{\phi}, \psi, \dot{\psi})^{T}$. Using this notation on the linear representation of the model in equation (11) one has

$$
\begin{array}{l|l|l}
\dot{x_{1}}=x_{2} & \dot{z_{1}}=z_{2} \\
\dot{x_{2}}=-z_{1} u_{1} & \dot{z_{2}}=u_{2} \\
\dot{x_{3}}=x_{4} & \dot{z_{3}}=z_{4} \\
\dot{x_{4}}=z_{3} u_{1} & \dot{z_{4}}=u_{3} \\
\dot{x_{5}}=x_{6} & \dot{z_{5}}=z_{6} \\
\dot{x_{6}}=1-u_{1} & \dot{z_{6}}=u_{4}
\end{array}
$$

\section{A. Operating modes of the road following mission}

The road following mission is divided in five stages. Stage 1 - Take-off mode: the objective is to achieve the desired altitude $z_{d}$. Stage 2 - $\psi$-alignment mode $(\psi \mathbf{A L})$ : once at the desired altitude, the task to be accomplished is to align the vehicle's heading angle w.r.t. the road direction. Stage 3 $y$-alignment mode $(y \mathbf{A L})$ : the vehicle is required to maintain a flying path well aligned w.r.t. the center of the road. Stage 4 - Navigation mode: the vehicle's forward displacement is stabilized in order to navigate at constant velocity. Stage 5 Landing mode: the altitude is controlled in order to perform an autonomous landing.

\section{B. Control Laws in each Operating Mode}

The control strategy proposed in all different modes is based on the idea that the system in equation (12) is constituted of two subsystems, the attitude dynamics and the position dynamics, existing a time-scale separation between them [11]. From this fact, it is possible to propose a hierarchical control scheme, where the positioning controller provides the reference attitude angles $\left(\theta_{d}, \phi_{d}\right.$ and $\left.\psi_{d}\right)$, which are the angles that must be tracked by the orientation controllers. Error dynamics of the model in equation (12) are represented by $\tilde{x}_{i}=x_{i}-x_{i_{d}}$ and $\tilde{z}_{i}=z_{i}-z_{i_{d}}$, with $i \in\{1, \ldots, 6\}$.

1) Attitude Control: An integral sliding mode control is proposed, which was chosen due to its insensitivity to model errors, parametric uncertainties and other disturbances [12]. For the pitch dynamics case, the error equation is defined as $\tilde{z}_{1}=z_{1}-z_{1_{d}}$. As shown in [13], lets select the switching function

$$
s(z, t)=\dot{\tilde{z}}_{1}+2 \lambda \tilde{z}_{1}+\lambda^{2} \int_{0}^{t} \tilde{z}_{1}(\tau) \mathrm{d} \tau
$$

which depends on the pitch dynamics states. The $\lambda$ parameter in equation (13) is the slope of the sliding line and should be greater than zero to ensure the asymptotic stability of the sliding mode. Computing the time derivative of equation (13) one has $\dot{s}=z_{1} u_{2}+2 \lambda z_{2}+\lambda^{2} \tilde{z}_{1}$. Considering the sliding mode condition $\dot{s}=0$, one finds the equivalent control $z_{1_{e q}}=$ $-2 \lambda z_{2}-\lambda^{2} \tilde{z}_{1}$. With the purpose of obtaining a control law such that the state vector $\tilde{z}_{1}$ remains on the sliding surface $s(z, t)=0, \forall t>0$, let's use the Lyapunov function candidate $v(s)=\frac{1}{2} s^{2}$. An efficient condition for the stability of the pitch sub-system can be satisfied if one can ensure that the reached condition $\dot{v}(s)=\frac{1}{2} \frac{d}{d t} s^{2} \leq \eta|s|$, with $\eta \geq 0$ holds. Then, the 
system remains on the sliding surface and the states go to the origin. Thus $s \dot{s} \leq-\eta|s|$ and the controller must be chosen such that $z_{1}=z_{1_{e q}}-K \operatorname{sign}(s)$, where $K$ is a positive real number. Following a similar approach, it is possible to obtain the yaw and roll angles controllers.

2) Position Control: Motion in the $x-y$ plane is accomplished by orientating the vehicle's thrust vector in the direction of the displacement desired. Therefore, the angles $\theta_{d}$ and $\phi_{d}$ act as virtual controllers for the position dynamics. The control laws proposed for the $z$ an $x$ positions, respectively, are expressed as

$$
\begin{aligned}
& u_{1}=k_{p z}\left(x_{5}-x_{5_{d}}\right)+k_{v z}\left(x_{6}-x_{6_{d}}\right)-1 \\
& \theta_{d}=\frac{k_{v x}\left(x_{2}-x_{2_{d}}\right)+k_{p x}\left(x_{1}-x_{1_{d}}\right)}{u_{1}}
\end{aligned}
$$

with $k_{v x}, k_{p x}, k_{p z}$ and $k_{v z}$ being positive real numbers.

The $y$-position control is composed by two PID controllers, one for the situation when the road is being detected and one for the situation when it is not. Although both PID controllers are similar, they have different gains. When the road is inside of the camera's FOV, the gains of the controller are tuned to behave like a PD controller, due to the necessity of having a rapid response for maintaining the vehicle's $y$ coordinate at a minimum value, i. e., $y=0$ [7]. On the other hand, when the vehicle looses the image of the line, a switch to a different method for measuring the vehicle's $\psi$ angle and $y$ position occur, and, at the same time, the positioning controller's gains switch to alternative values simulating a PI controller. In both of them, the control objective is to regulate the $x_{3}$ state to the origin, i.e. $x_{3 d}=0$.

For the case when the line is detected, the feedback control law proposed is given by

$$
z_{3}=\frac{1}{u_{1}}\left(-k_{L 3} x_{3}-k_{L 4} x_{4}+k_{L I} \xi\right)
$$

where $k_{L 3}, k_{L 4}$ and $k_{L I}$ are positive real numbers. It has been introduced the additional state $\xi$, whose dynamics are given by $\dot{\xi}=x_{3 d}-x_{3}=-x_{3}$. Using the control in equation (16), the closed-loop system of the lateral dynamics is given as

$$
\begin{aligned}
& \dot{x}_{3}=x_{4} \\
& \dot{x}_{4}=-k_{L 3} x_{3}-k_{L 4} x_{4}-k_{L I} \xi \\
& \dot{\xi}=-x_{3}
\end{aligned}
$$

Equation (17) can be represented as $\dot{e}_{y}=A_{L} e_{y}$, where $e_{y}=$ $\left(x_{3}, x_{4}, \xi\right)^{T}$ and

$$
A_{L}=\left(\begin{array}{ccc}
0 & 1 & 0 \\
-k_{L 3} & -k_{L 4} & k_{L I} \\
-1 & 0 & 0
\end{array}\right)
$$

For the case when the line is not detected, the control schema proposed is the same as was given by equation (16), with the only difference of having a set of different gains. Then, the closed-loop system is represented as $\dot{e}_{y}=A_{N L} e_{y}$, where

$$
A_{N L}=\left(\begin{array}{ccc}
0 & 1 & 0 \\
-k_{N L 3} & -k_{N L 4} & k_{N L I} \\
-1 & 0 & 0
\end{array}\right)
$$

whit $k_{N L 3}, k_{N L 4}$ and $k_{N L I}$ being positive real numbers. Remark 1: On the experimental platform, the gains were tuned experimentally, resulting in different values for both cases. In the first approach (road detected), they were selected as $K_{L 3}=1000, K_{L 4}=850$ and $K_{L I}=0.1$, while in the second one (no road detected) as $k_{N L 3}=1000, k_{N L 4}=1$ and $k_{N L I}=9.5$. In this way, the system presents a switchedsystem characteristic.

\section{Stability Analysis of the Lateral Position Control}

This section focuses on the system's stability across switching boundaries, i.e., where the control gains of equation (16) switch to different values. Following a similar approach than the one presented in [14], it is possible to find a common Lyapunov function for the closed-loop system formed from applying the two controllers of the lateral position dynamics. However, working in this way, the same pole locations have to be chosen for both cases, which in fact is not the case (different gain values are being applied).

Let's define $d_{c}$ as the distance measured from the vehicle's center of gravity projection to the point where the camera loses the image of the road, see Fig. 1. Thus, a change of coordinates can be made such that $y_{d_{1}}=x_{3}+d_{c}$ and $\dot{y}_{d_{1}}=y_{d_{2}}=$ $x_{4}$ is its derivative. From equation (17) one has $\dot{y}_{d_{1}}=y_{d_{2}}$, $\dot{y}_{d_{2}}=-k_{L 3} y_{d_{1}}-k_{L 4} y_{d_{2}}-k_{L I} \xi, \dot{\xi}=-y_{d_{1}}$, with $e_{y_{d}}=$ $\left(y_{d_{1}}, y_{d_{2}}, \xi\right)^{T}$. It can be defined a state-dependent switched linear system, given by the closed-loop system together with the switching conditions

$$
\dot{e}_{y_{d}}=\left\{\begin{array}{cc}
A_{L} e_{y_{d}} & \text { if }\left|y_{d_{1}}\right|<0 \\
A_{N L} e_{y_{d}} & \text { if }\left|y_{d_{1}}\right| \geq 0
\end{array}\right.
$$

It is clear that each individual system in equation (20) is stable, since the matrices $A_{L}$ and $A_{N L}$ are Hurwitz. Suppose that there is a family $A_{p}, p \in \mathcal{P}$ of functions from $\Re^{n}$ to $\Re^{n}$, with $\mathcal{P}=1,2, \ldots, m$ defining the finite index set. For the case of linear systems, this results in a family of systems $\dot{x}=A_{p} x$ with $A_{p} \in \Re^{n \times n}$. Let's define a piecewise constant function $\sigma:[0, \infty) \rightarrow \mathcal{P}$ with finite number of discontinuities (switching times) on every bounded time interval. This function takes a constant value on every interval between two consecutive switching times. Then $\sigma$ gives the index $\sigma(t) \in \mathcal{P}$ of the system that is actually active, at each instant of time $t$.

Theorem 1: Consider vectors $t_{p q}$, symmetric matrices $S_{p}$ with $\Omega_{p} \in\left\{x: x^{T} S_{p} x \geq 0\right\}, \forall p \in \mathcal{P}$ having non-negative entries and symmetric matrices $P_{p}$ such that:

$$
\begin{array}{r}
A_{p}^{T} P_{p}+P_{p} A_{p}+\beta_{p} S_{p}<0, \quad \beta_{p} \geq 0 \\
0<P_{p}-P_{q}+f_{p q} t_{p q}^{T}+t_{p q} f_{p q}^{T} \text { for some } t_{p q} \in \Re^{n}
\end{array}
$$

With the boundary between $\Omega_{p}$ and $\Omega_{q}$ of the form $\left\{x: f_{p q}^{T}=\right.$ $0\}, f_{p q} \in \Re^{n}$. Then every continuous, piecewise $\mathcal{C}^{1}$ trajectory of the system $\dot{x}=A_{\sigma} x$ tends to zero exponentially.

Proof: See the Appendix.

\section{EXPERIMENTAL PLATFORM}

The quad-rotor has a dimension of $40 \mathrm{~cm}$ between same axis rotors, a weight of $1.1 \mathrm{~kg}$. and an autonomy of $15 \mathrm{~min}$. Control 
electronics is based on a IGEPv2 card equipped with a $\mathrm{TI}^{\circledR}$ DM3730 System On Chip where the control law is executed at a $100 \mathrm{~Hz}$. A Microstrain ${ }^{\circledR}$ IMU provides measurements at $100 \mathrm{~Hz}$ while an SRF10 ultrasonic sensor measures the altitude between 0 and $2 \mathrm{~m}$. Images are provided at $120 \mathrm{~Hz}$ by a PlayStation ${ }^{\circledR}$ Eye camera pointing downwards. The Harris affine region detector is implemented to perform characteristic features detection. The optical flow algorithm implemented is based on the pyramidal Lucas-Kanade method. The computation of the vehicle's heading angle and lateral position are based on a Hough transform technique for detecting lines. The imaging algorithms are based on openCV functions [15] and are executed in the onboard DSP at $100 \mathrm{~Hz}$. The quad-rotor is wirelessly linked to a ground station PC where a graphical user interface allows monitoring and controlling the vehicle. The quad-rotor can be controlled manually by means of a PlayStation ${ }^{\circledR} 3$ joystick, which is linked with BlueTooth ${ }^{\mathrm{TM}}$ to the ground station.

\section{REAL TIME EXPERIMENTS}

The road following mission in presence of external disturbances was performed autonomously by the quad-rotor over a road model having two smooth curves. The experiment procedure is just as explained in Section II. An external disturbance is applied during navigation which makes the imaging system unable to detect the road, this has the purpose of demonstrating the effectiveness of the estimators and controllers during switching times. A video of the road following experiment can be seen at

http://youtu.be/IV75kaMt_gg

The quad-rotor translational dynamics are shown in Fig. 2. From the upper graphic, $x=10$ meters represents the longitudinal distance flown by the quad-rotor, obtained by integrating the optical flow sensed in the $x$ direction. The $y$ parameter represents the lateral position of the quad-rotor w.r.t. the road. It can be seen an external perturbation induced in the $y$ direction at $t=26$ seconds. The lower graphic represents the vehicle's altitude. Translational velocities are shown in Fig. 3. Notice that the forward speed $\dot{x}$ is maintained at $1 \mathrm{~m} / \mathrm{s}$, while $\dot{y}$ and $\dot{z}$ are kept near zero.

The switching between the estimation approaches is shown in Fig. 4. The middle graphic represents the time instants where the line is being detected $(s=1)$ and where it is not $(s=0)$. The upper graphic represents the desired heading angle that will align the vehicle heading with the road direction, which is computed from equation (9). The lower graphic represents the lateral position error as computed from equation (10). A zoom of the region where the switch occurs $(t=26 \mathrm{~s})$ is shown in Fig. 5.

The helicopter's Euler angles, as obtained directly from the embedded IMU are shown in Fig. 6. Angles $\theta$ and $\phi$ are always kept small while performing the mission. The lower graphic shows the heading angle described by the vehicle in order to handle the two road's curves. This heading angle is represented
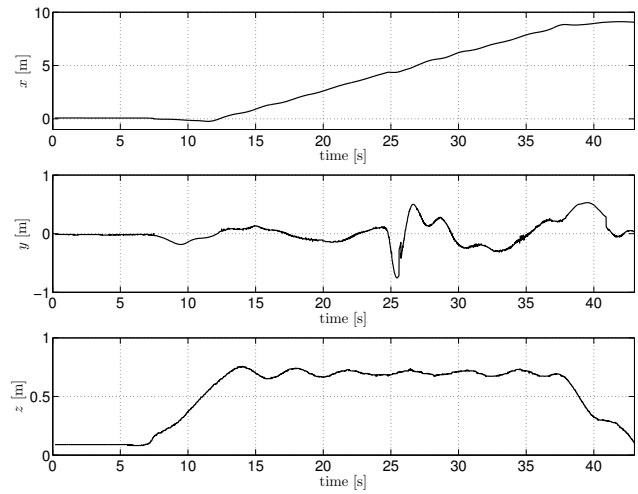

Fig. 2. $(x, y, z)$ states. Perturbation in $y$ position is shown at $t=26 \mathrm{~s}$.
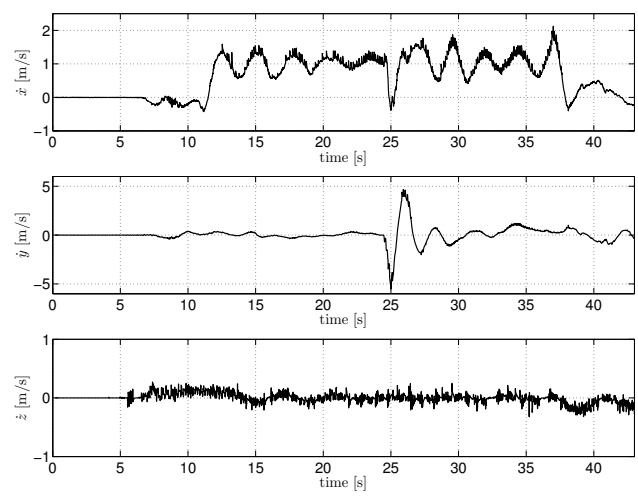

Fig. 3. Translational velocities during the experiment.
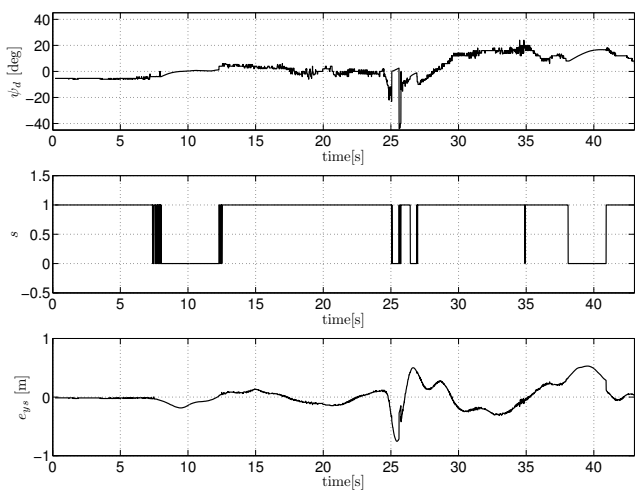

Fig. 4. Switching signals: $\psi_{d}$, switch signal $s, e_{y s}$.

w.r.t. the inertial reference frame whose origin is at the quadrotor initial position.

\section{CONCLUSiON}

The problem of vision-based road following using a quadrotor was addressed. The goal of this research consisted on estimating and tracking a road without a priori knowledge of the path to be tracked, as well as of deriving efficient 

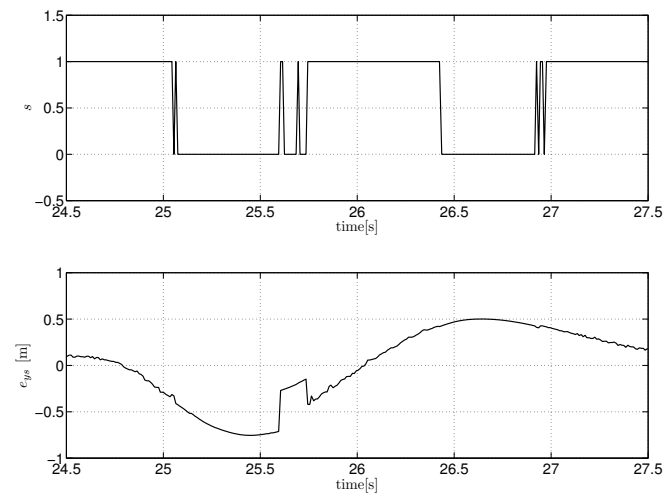

Fig. 5. Zoom of signal $s$ affecting the lateral position measurement method.
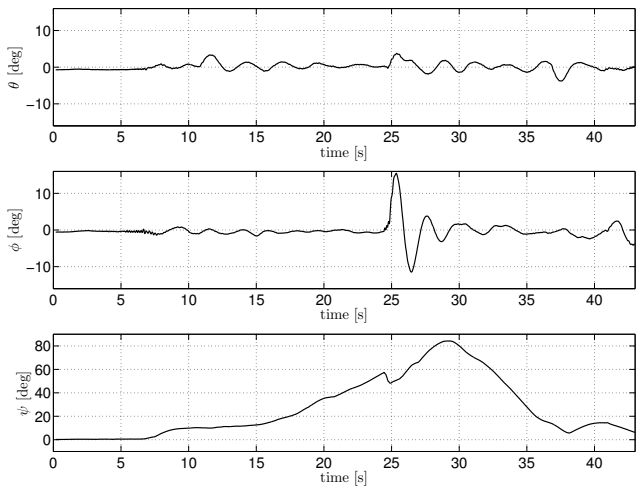

Fig. 6. The helicopter Euler angles during flight.

controllers for dealing with situations when the road is not detected in the camera's image. Two operational regions were defined: one for the case when the road is detected and the other one for when it is not. A switching between imaging and inertial sensors measurements was used for estimating the vehicle's parameters in both regions. For dealing with both aforementioned cases a switching control strategy which stabilizes the vehicle's lateral position was proposed. The system's stability was proved not only in the two regions, but also in the switching boundaries between them. The performance of the switching control was tested in real time experiments, demonstrating the effectiveness of the proposed approach for performing the task of road following in the presence of external disturbances.

Future work will consider a hybrid scheme for switching not only different gains but also different control strategies.

\section{APPENDIX}

Before proving Theorem 1, let's use the following theorem.

Theorem 2: The system $\dot{x}=f(t, x), f(t, x) \equiv 0$, is exponentially stable on the region $D=\left\{x \in \Re^{n} \mid\|x\|<r\right\}$ if there exists a Lyapunov function $V(t, x)$ and some positive constants $c_{1}, c_{2}, c_{3}$, such that $\forall(t, x) \in[0, \infty) \times D_{0}$,

$$
\begin{aligned}
& D_{0}=\left\{x \in \Re^{n} \mid\|x\|\right.<r / m\} \\
& c_{1}\|x\|^{2} \leq V(t, x) \leq c_{2}\|x\|^{2} \\
& \frac{\partial V}{\partial t}+\frac{\partial V}{\partial x} \leq-c_{3}\|x\|^{2}
\end{aligned}
$$

where $m$ is the overshot from definition of exponential stability.

Proof: See [16], pp. 169.

Proof of Theorem 1: The proof relies on Theorem 2, then using the Lyapunov function candidate $V(x)=x^{T} P_{p} x$ and assuming that $x(t)$ is continuous and piecewise $\mathcal{C}^{1}$, hence, $V(t)$ has the same characteristics. Premultiplying and postmultiplying condition (22) by $x$, the inequality on the left side of (23) is satisfied. Similarly, inequality (24) follows if we premultiply and postmultiply both sides of (21) by $x$.

\section{REFERENCES}

[1] E. Altug, J. Ostrowski, and R. Mahony, "Control of a quadrotor helicopter using visual feedback," in International Conference on Robotics and Automation, Washington, DC, May, 2002, pp. 72-77.

[2] S. Zingg, D. Scaramuzza, S. Weiss, and R. Siegwart, "Mav navigation through indoor corridors using optical flow," in International Conference on Robotics and Automation, Anchorage, Alaska, 2010.

[3] D. Cabecinhas, C. Silvestre, and R. Cunha, "Vision-based quadrotor stabilization using a pan and tilt camera," in Conference on Decision and Control, Atlanta, GA, USA, December, 2010, pp. 1644-1649.

[4] L. García-Carrillo, E. Rondón, A. Sánchez, A. Dzul, and R. Lozano, "Stabilization and trajectory tracking of a quad rotor using vision," Journal of Intelligent and Robotic Systems, vol. 61, no. 1-4, pp. 103118, January, 2011.

[5] E. Rondon, L. García-Carrillo, and I. Fantoni, "Vision-based altitude, position and speed regulation of a quad-rotor rotorcraft," in International Conference on Intelligent Robots and Systems, Taipei, Taiwan, October, 2010 , pp. $628-633$.

[6] J. Hespanha, O. Yakimenko, I. Kaminer, and A. Pascoal, "Linear parametrically varying systems with brief instabilities: An application to integrated vision/imu navigation," IEEE Transactions on Aerospace and Electronic Systems, vol. 40, no. 3, pp. 889-902, July, 2004.

[7] G. Hoffmann, H. Huang, S. L. Waslander, and C. Tomlin, "Quadrotor helicopter flight dynamics and control: Theory and experiment," in Proc. of the AIAA Guidance, Navigation, and Control Conference, Hilton Head, South Carolina, USA, 2007.

[8] M. Oishi and C. Tomlin, "Switching in nonminimum phase systems: Application to vstol aircraft," in Proc. IEEE American Control Conference (ACC'2000), Chicago, IL, Jun. 2000, pp. 838-843.

[9] F. Chaumette, "Image moments: a general and useful set of features for visual servoing," IEEE Trans. on Robotics, vol. 20, no. 4, pp. 713-723, August, 2004.

[10] L. García-Carrillo, A. Dzul, R. Lozano, and C. Pégard, "Combining stereo vision and inertial navigation system for a quad-rotor uav," Journal of Intelligent and Robotic Systems, vol. DOI: 10.1007/S10846011-9571-7, April, 2012.

[11] P. Kokotović, H. K. Khalil, and J. O'Reilly, Singular Perturbation Methods in Control: Analysis and Design. London: Academic Press: Siam, 1999.

[12] R. Xu and U. Ozguner, "Sliding mode control of a class of underactuated systems," Automatica, vol. 44, no. 1, pp. 233-241, January, 2008.

[13] J. Slotine and W. Li, Applied Nonlinear Control. Prentice Hall, 1990.

[14] D. Liberzon, Switching in Systems and Control. Boston: Birkhuser, 2003.

[15] G. Bradski and A. Kaehler, Learning OpenCV. O'Reilly Media, September 2008.

[16] H. K. Khalil, Nonlinear Systems. New York: Prentice Hall, 2002. 